Conclusion The algorithm showed good performance to identify COPD patients among those individuals registered in the regional healthcare system confirming the strength of administrative data for monitoring chronic diseases.

\section{P2-142 EFFECT OF CHRONIC THERAPY WITH STATINS BEFORE INFARCTION IN PATIENTS WITH ACUTE MYOCARDIAL INFARCTION}

doi:10.1136/jech.2011.142976i.77

${ }^{1} \mathrm{C}$ Sorge, ${ }^{1} \mathrm{U}$ Kirchmayer, ${ }^{*}{ }^{1} \mathrm{~A}$ P Barone, ${ }^{1} \mathrm{~N}$ Agabiti, ${ }^{1} \mathrm{D}$ Fusco, ${ }^{1} \mathrm{~V}$ Belleudi, ${ }^{1} \mathrm{M}$ Davoli, ${ }^{2} \mathrm{C}$ A Perucci. ${ }^{1}$ Department of Epidemiology of the Regional Health Service, Lazio Region, Italy; ${ }^{2}$ National Agency of Regional Health Services, Roma, Italy

Introduction Efficacy of preventive therapy with statins in reducing mortality and cardiovascular morbidity in patients with and without cardiovascular disease has been shown in clinical settings, but effectiveness results were not reported.

Objectives To evaluate whether chronic statin therapy before infarction reduces mortality within 30 days after acute myocardial infarction (AMI) and investigate potential confounders/effect modifiers (gender, age and comorbidities).

Methods Hospital discharges of AMI patients ( $\geq 35$ years) resident in the Lazio region in 2007-2008, were selected, excluding patients with AMI or revascularisation procedures (aortocoronary bypass, PTCA) in the preceding 9 years. Statin use (ATC: C10AA, C10B) during 1 year preceding the index admission was assessed through the drug claims information system; patients were defined "partially adherent" with $20 \%-80 \%$ days covered by statin doses and "adherent" with $80 \%$ and more coverage. Crude and adjusted 30-day mortality risks of statin users vs nonusers were estimated using logistic regression models. The role of gender and age as effect modifiers, interaction terms were included in the model.

Results Among 6790 AMI patients statin users showed reduced mortality risk compared to nonusers ( $\mathrm{OR}=0.75,95 \%$ CI 0.58 to 0.98 for partially adherent, $\mathrm{OR}=0.8595 \% \mathrm{CI} 0.66$ to 1.09 for adherent). The protective effect was more evident in females $(\mathrm{OR}=0.74,95 \%$ CI 0.52 to 1.05 for partially adherent, $\mathrm{OR}=0.6795 \% \mathrm{CI} 0.46$ to 0.97 for adherent) and in older people ( $>85$ years) $(\mathrm{OR}=0.84,95 \% \mathrm{CI}$ 0.50 to 1.40 for partially adherent, $\mathrm{OR}=0.5195 \% \mathrm{CI} 0.29$ to 0.91 for adherent).

Conclusions Chronic statin therapy before infarction reduced 30 days mortality in patients with AMI, especially in females and elderly.

\section{P2-143 TRENDS IN THE PREVALENCE OF DIABETES AND DIABETIC RETINOPATHY AMONG JAPANESE URBAN POPULATION}

doi:10.1136/jech.2011.142976i.78

A Kitamura, ${ }^{*}$ E Nagano, M Uno, M Okada, M Kiyama, M Nakamura, T Okada, K Maeda, Y Shimizu, Y Ishikawa. Osaka Medical Center for Health Science and Promotion, Osaka, Japan

Introduction The purpose of this study was to examine trends in the prevalence of diabetes (DM) and diabetic retinopathy (DR) between 2001 and 2009 years among Japanese population in Osaka, urban area in Japan.

Methods This study was a population-based study including 10 companies' employees and 1 community residents in Osaka, aged in 30-79 years, who underwent cardiovascular routine health examination at 2001-2003 (7186 participants), 2004-2006 (6908 participants) and 2007-2009 (7530 participants). We compared the prevalence of DM and DR among these three periods. DM was defined as $\mathrm{HbA1} \geq 2.5 \%$ (Japan Diabetes Society's unit) or patients taking medication, and pre-DM was defined as $\mathrm{HbA1c} \geq 5.6 \%$ and $\mathrm{HbA} 1 \mathrm{c}<6.5 \%$. Fundus photographs were graded using modified Scott classification of diabetic change. DR was defined as 1 degree or greater of Scott classification or as significant retinal haemorrhage excluding hypertensive retinal haemorrhage and retinal vein thrombosis.

Results Age-adjusted prevalence of DM was also significantly increased from 2001 to 2003 (2.6\%) to 2007-2009 (3.9\%) in men and women ( $\mathrm{p}$ for trend $<0.01$ ). The respective prevalence of preDM was also significantly increased from $3.9 \%$ to $8.7 \%$ (p for trend $<0.001)$. The age-adjusted prevalence of DR was $13 \%-15 \%$ in men and $11 \%-13 \%$ in women, both of which showed no significant change from 2001-2003 to 2007-2009.

Conclusion A significant increase in the prevalence of DM and preDM implies the importance of prevention and early detection for DM and DR in urban Japanese populations.

\section{P2-144 AN EVALUATION OF DIETARY AND EXERCISE INTERVENTION FOR HYPERTENSIVE MEN}

doi:10.1136/jech.2011.142976i.79

${ }^{1} \mathrm{~K}$ Kitaoka, ${ }^{1,2} \mathrm{~J}$ Nagaoka, ${ }^{1,3} \mathrm{~T}$ Matsuoka, ${ }^{1,4} \mathrm{C}$ Shigemura, ${ }^{1} \mathrm{~W}$ Aoi, ${ }^{1} \mathrm{~S}$ Wada, ${ }^{5} \mathrm{H}$ Asano, ${ }^{6} \mathrm{~N}$ Sakane, ${ }^{1} \mathrm{~A}$ Higashi. ${ }^{1}$ Graduate School of Life and Environmental Sciences, Kyoto Prefectural University, Kyoto, Japan; ${ }^{2}$ Higashiosaka City, Osaka, Japan; ${ }^{3}$ Kasai City, Hyogo, Japan; ${ }^{4}$ Kyoto College of Nutritional \& Medical Sciences, Kyoto, Japan; ${ }^{5}$ School of Nursing, Kyoto Prefectural University of Medicine, Kyoto, Japan; ${ }^{6}$ Division of Preventive Medicine, Clinical Research Institute, National Hospital Organization Kyoto Medical Center, Kyoto, Japan

Introduction This study evaluated the effect of a 6-month health intervention program conducted from 2003 to 2008 for hypertensive men in Japan.

Methods The subjects, volunteers aged $50-75$ years, were divided into 45 and 25 men for the intervention and control groups, respectively. We performed dietary and exercise education five times for the intervention group and conducted two health checkups for both groups. We compared lifestyle, and physical and mental health criteria at baseline, and immediately after the intervention.

Results During the 6-month intervention, the follow-up rates were $97.8 \%$ and $76.0 \%$ in the intervention and control groups, respectively. At the baseline, there were no differences in physical status or lifestyles between the intervention and control groups. After the program, no significant change was shown in the urinary excretion ratio of $\mathrm{Na} / \mathrm{K}$ in the control group $(3.5-3.2, p=0.768$ ), but a significant decrease was demonstrated in the intervention group $(2.6-2.2, \mathrm{p}=0.023)$. The exercise habit, a walk of more than $30 \mathrm{~min}$ everyday, increased significantly in the intervention group. No significant change in the mean systolic or diastolic blood pressure $(\mathrm{mm} \mathrm{Hg})$ was shown in the control group (149.3-147.0, $\mathrm{p}=0.199$, $89.2-87.5, p=0.171)$, but the intervention group showed a significant decrease $(151.0-146.0, p=0.034,93.5-88.6, p<0.001)$.

Conclusion In the intervention group, dietary and exercise habits were improved by health education, and the $\mathrm{Na} / \mathrm{K}$ ratio and blood pressure significantly decreased for the next 1 -year period.

\section{P2-145 BMI AND THE RISK OF HOSPITALISATION}

doi:10.1136/jech.2011.142976i.80

${ }^{1} \mathrm{R}$ Korda, ${ }^{* 1} \mathrm{E}$ Banks, ${ }^{1} \mathrm{M}$ Clements, ${ }^{2} \mathrm{~A}$ Bauman, ${ }^{3,4} \mathrm{~B}$ Liu, ${ }^{3} \mathrm{H}$ Bambrick, ${ }^{3} \mathrm{~L}$ Jorm. ${ }^{1}$ The Australian National University, Canberra, ACT, Australia; ${ }^{2}$ University of Sydney, Sydney, New South Wales, Australia; ${ }^{3}$ University of Western Sydney, Sydney, New South Wales, Australia; ${ }^{4}$ University of NSW, Sydney, New South Wales, Australia

Introduction Obesity and overweight have a range of health effects, yet little evidence is available on their effect on hospitalisation. 